\title{
A Motivação de Adolescentes em Relação com a Perspectiva de Tempo Futuro
}

\author{
The Motivation of Adolescents in Relation to the Perspectives of the Future \\ Adriana Cristine Dias Locatelli*, José Aloyseo Bzuneck \& Sueli Édi Rufini Guimarães \\ Universidade Estadual de Londrina, Paraná, Brasil
}

\begin{abstract}
Resumo
O objetivo do presente trabalho foi verificar se adolescentes $(\mathrm{N}=2 \mathrm{O} 6)$ percebem os estudos atuais como meios para atingirem metas profissionais futuras. Os dados foram levantados por meio de um questionário em escala Likert. Inicialmente, identificaram-se dois grupos, dos já definidos e daqueles que ainda não se definiram quanto à profissão futura, e que foram comparados em relação à motivação, ao uso de estratégias de estudos (gerenciamento de tempo) e à percepção de instrumentalidade dos estudos atuais. Os resultados revelaram diferenças significativas entre os dois grupos. E, entre os já definidos, a perspectiva de tempo futuro apareceu mais associada à motivação e ao uso de estratégias e com valor preditivo da percepção de instrumentalidade. Os resultados foram discutidos à luz de teorias motivacionais, com implicações para o trabalho dos professores em relação à motivação de adolescentes.

Palavras-chave: Motivação de adolescentes; perspectiva de tempo futuro; definição vocacional na adolescência.
\end{abstract}

\begin{abstract}
The aim of the present study was to verify if adolescents $(\mathrm{N}=206)$ perceive their current academic tasks as means to accomplish their professional goals in the future. Data were collected through a Likert scale questionnaire. Initially, two groups were identified, those with a defined future professional goal and those that have not yet decided. Both groups were compared regarding motivation, study strategies (time management) and perception of present schooling as instrumental to attaining their future goals. Results showed some significant differences between groups. Among those with a defined future goal, their perspectives on the future were more associated to motivation, the use of strategies and perception of instrumentality. Regression analysis showed some important predictive values. Results were discussed in the light of modern motivational theories, with implications leading to the work of teachers in relation to the motivation of adolescents.

Keywords: motivation of adolescents; perspectives of the future; vocational decisions in adolescence..
\end{abstract}

Motivar adolescentes para os estudos tem sido considerada tarefa particularmente desafiadora para professores e educadores, dadas as condições contextuais e as características dos próprios alunos nessa faixa etária (Zusho \& Pintrich, 2001; para o caso brasileiro, conferir a revisão de Bzuneck \& Boruchovitch, 2003). A literatura mais recente apresenta as variáveis que as pesquisas têm identificado e que nos fornecem uma compreensão de como motivar alunos em geral. Assim, a motivação tem sido estudada em associação com as metas de realização (ver, por exemplo, Pintrich, 2000), com as atribuições de causalidade (Weiner, 1984, 2004), em termos de motivações intrínseca ou extrínseca (Deci \& Ryan, 1985; Ryan \& Deci, 2000), de auto-eficácia (Bandura, 1997), para citar apenas as abordagens mais freqüentemente adotadas nas pesquisas contemporâneas e que focalizam fatores motivacionais próximos do aluno na escala de tempo.

Quando se considerou a fase da adolescência como objeto de estudo em relação com escolaridade, desenvolveu-se,

*Endereço para correspondência: Rua Lourdes Martins Martinelli, 170, Londrina, Paraná, 86035-176. Fone: 3371- 4338. E-mail: adrimar@sercomtel.com.br além dessas abordagens, uma vertente cognitivista que considera a motivação em função da perspectiva do tempo futuro, ou seja, dos objetivos de vida que a pessoa se propõe atingir. Na adolescência, etapa caracterizada por diversas transformações que marcam a transição entre a meninice e a idade adulta, a pessoa se torna capaz de estabelecer de modo realístico um projeto de vida (Lens, Simons \& Dewitte, 2002), o que é ao mesmo tempo uma tarefa determinada socioculturalmente. Espera-se que, ao menos pelo final desse período e como ingrediente da formação de sua identidade pessoal, os indivíduos se tenham decidido sobre o próprio futuro, particularmente em termos profissionais. Tal perspectiva do tempo futuro consiste no estabelecimento de metas de vida, também denominadas de tarefas de vida, projetos pessoais ou possíveis eus "possible selves" (Brickman \& Miller, 2001; MarKus \& Nurius, 1976). No cumprimento dessa exigência psicossocial o adolescente recebe influências múltiplas da família, de colegas, da escola e da mídia, que podem representar tanto contribuições positivas como fatores de maior perplexidade e indecisão. Além disso, a formação da perspectiva de tempo futuro é moderada por 
variáveis como idade cronológica, nível sócio-econômico e também possivelmente inteligência (Lens et al., 2002). Por esses motivos, ao final do ensino médio, um(a) jovem pode já ter definido suas metas de vida, como também poderá estabelecê-las somente após um período mais ou menos prolongado de incertezas, de busca e de experiências.

A questão básica que se levanta é a seguinte: o fato de um(a) adolescente já ter configurado seu projeto de profissão futura constitui-se em fator motivacional para os estudos no presente? Em outras palavras, qual a relação mais precisa entre a motivação atual dos alunos e o seu projeto de vida? Neste sentido, as descobertas de pesquisa propiciam ou sugerem estratégias de ensino para motivar adolescentes recorrendo-se a seus objetivos de vida. Estas foram as questões que basicamente nortearam a presente pesquisa.

Para uma compreensão inicial do significado de metas futuras para a motivação no presente, deve-se levar em conta que a Perspectiva de Tempo Futuro tem sido conceituada como a antecipação no presente de metas futuras, ou seja, diz respeito ao grau e ao modo pelo qual o futuro cronológico de um indivíduo é integrado ao espaço de vida presente através de processos motivacionais (Husman \& Lens, 1999; Lens et al., 2002; Miller \& Brickman, 2004). Tais metas futuras podem ser relativamente próximas (como passar no vestibular) ou bem distantes no tempo (como sucesso numa determinada profissão). Em qualquer caso, como o explicitam Miller e Brickman (2004; ver também Husman \& Lens, 1999), o fator motivacional para o envolvimento com as tarefas escolares no presente deriva da valorização da meta futura e, simultaneamente, da percepção de que as tarefas escolares atuais são instrumento ou meio para a consecução daquela meta.

De Volder e Lens (1982) esclarecem ainda que a perspectiva do tempo futuro não se reduz a uma simples fantasia quanto ao futuro. Propuseram que nessa perspectiva se encontram dois aspectos distintos, o dinâmico e o cognitivo, uma distinção posteriormente retomada por Husman e Lens (1999). O aspecto dinâmico é a disposição para se valorizar uma determinada meta distante, ou seja, essa meta futura aparece como importante, merecedora de ser perseguida, embora longínqua. Já o aspecto cognitivo refere-se à percepção de que as ações presentes têm o valor de instrumentalidade, ou seja, conta com a capacidade do indivíduo antecipar no presente não somente as conseqüências imediatas de uma ação, mas também as de longo alcance. A literatura atesta que os alunos que formaram uma perspectiva de tempo futuro longa podem antecipar de modo mais fácil as implicações de suas tarefas presentes para alcançar suas metas futuras. Em seu estudo com adolescentes, De Volder e Lens (1982) descobriram que tanto o desempenho por notas como a persistência nos estudos estavam positivamente relacionados com alta valorização de metas num futuro distante. Isto é, a percepção do valor de instrumentalidade ou utilidade das ações presentes incrementam a motivação para aprender e realizar as tarefas na escola (ver também Van Calster, Lens \& Nuttin, 1987).
Nesse contexto de valorização das metas futuras como das ações presentes enquanto instrumentos, alguns autores mais recentes (Husman \& Lens, 1999; Malka \& Covington, 2005; Simons, Vanteenkiste, Lens \& Lacante, 2004) têm feito recurso à teoria de expectativa-valor desenvolvida por Eccles, Wigfield e pesquisadores (ver descrição completa em Eccles \& Wigfield, 2002; Wigfield \& Eccles, 2000; Wigfield, Tonks \& Eccles, 2004). De acordo com essa teoria, a crença sobre o valor subjetivo de uma dada tarefa engloba quatro componentes: o interesse intrínseco, a utilidade, a importância e o custo. O aspecto de interesse intrínseco configura-se quando a pessoa sente prazer na realização da tarefa; a utilidade refere-se ao grau em que a pessoa julga que a tarefa é meio para realizar algum plano futuro; a importância, ligada a aspectos da identidade, significa que o cumprimento de uma tarefa é visto como central no atendimento de necessidades pessoais como de poder, afiliação, entre outras; e o custo refere-se a esforço e possíveis sacrifícios para executar a tarefa. Como se pode facilmente depreender, em termos motivacionais, a percepção da relação entre os estudos atuais e a perspectiva do tempo futuro carrega mais no componente utilidade. E a própria meta futura será valorizada em função de um ou mais dentre esses quatro componentes. Pesquisas empíricas têm dado apoio e prestado esclarecimentos significativos a essas proposições.

\section{Estudos Empíricos}

Van Calster, Lens e Nuttin (1987), em seu estudo com 230 alunos com idade entre 17 e 19 anos, concluíram que quando eles percebem a escolarização como importante para o sucesso no futuro são significativamente mais motivados do que aqueles que a percebem como menos importante. Em poucas palavras, a motivação escolar é afetada pela percepção de instrumentalidade. Entretanto, os autores também descobriram que a atitude afetiva para com o próprio futuro é uma variável crucial para a percepção de instrumentalidade. Isto é, quando o aluno tem alta percepção de instrumentalidade de que fazer o melhor na escola conduz a um futuro pessoal, juntamente com uma atitude positiva para com o futuro, é maior a motivação e são melhores os resultados acadêmicos. Ao contrário, uma atitude muito negativa quanto ao futuro pessoal torna-se fator de desmotivação. A atitude afetiva dos alunos em relação ao futuro apareceu também como variável crítica da motivação no estudo de Lens e Decruynaere (1991).

Zusho e Pintrich (2001), ao comentarem o declínio de interesse pelas atividades acadêmicas na adolescência, apontam que estes começam a apresentar um interesse individual por atividades específicas, como matemática, ciências ou atletismo, de acordo com as preferências e valorizações próprias. De modo geral, segundo esses autores, os alunos do ensino fundamental são mais ligados aos trabalhos escolares do que os do ensino médio, mas não se pode afirmar que não haja algum interesse por 
parte destes últimos. Ocorre que estes são mais seletivos quanto à maneira de usar seu tempo e podem definir de modo mais independente seus interesses acadêmicos.

Em uma pesquisa realizada na Bélgica, Cretens, Lens e Simons (2001) procuraram verificar o papel da percepção de instrumentalidade em uma amostra de 733 participantes de uma escola vocacional, que corresponderia aproximadamente a algum tipo de ensino técnico aqui no Brasil. Os autores haviam constatado que nas escolas regulares de ensino médio havia sérios problemas quanto à motivação, principalmente em matérias teóricas, tais como matemática, história e línguas. Neste estudo, os autores procuraram verificar se os alunos que percebiam a relevância de uma segunda língua para o futuro (no caso, o francês), estavam mais motivados para este curso do que os alunos que não viam tal relevância. Surgiram correlações significativas entre instrumentalidade e motivação para metas próximas e distantes. Tanto em relação a metas próximas quanto distantes, alunos com baixa percepção de instrumentalidade apresentaram uma motivação significativamente menor para os estudos. Quanto à utilidade das disciplinas, os alunos que descreveram mais utilidade em seus cursos práticos estavam mais motivados para estas disciplinas do que para as disciplinas mais teóricas ou mesmo para o francês como uma segunda língua, apesar de atribuírem alto valor de utilidade para o francês.

Diante dos resultados, os autores concluíram que a percepção de instrumentalidade é um critério importante, mas não uma condição suficiente para o entendimento e desenvolvimento da motivação acadêmica, já que fatores mais específicos, como questões culturais, também contribuem. Para Husman e Lens (1999), apenas a percepção de instrumentalidade não é capaz de sustentar o interesse em conteúdos acadêmicos, sendo somente uma parte do grande quebra-cabeça que é a motivação do aluno para aprender.

Por outro lado, mesmo quando a meta futura for pessoalmente valorizada, sendo então incentivo para comportamentos instrumentais, é a percepção dos estudos presentes como meios para se chegar à meta valorizada que apareceu associada a mais esforço, tempo despendido e resultados por notas. Isto foi descoberto em estudos com alunos de cursos superiores por Malka e Covington (2005) e Shell e Husman (2001).

O recente estudo de Greene, Miller, Crowson, Duke e Akey (2004) com alunos do ensino médio trouxe importantes descobertas em relação à percepção dos estudos atuais como instrumentais para as metas de vida dos alunos. Os resultados indicaram que as percepções das tarefas escolares como significativas, relevantes e interessantes, ou seja, positivamente motivadoras, influenciam nas percepções de que essas tarefas são instrumentais para seus propósitos futuros. Na relação entre a percepção de instrumentalidade para o futuro e a adoção da meta de realização denominada domínio ou aprender, descobriu-se que quando os alunos percebem que as aprendizagens têm hoje o significado de proporcionar aumento de conhecimentos e habilidades, relevantes para o sucesso no futuro, eles também tendem a adotar mais freqüentemente a meta domínio.

Em síntese, a literatura tem demonstrado, particularmente em relação a adolescentes, que perceber um comportamento presente como meio ou instrumento para se atingir objetivos localizados num futuro próximo ou distante pode despertar e manter a motivação escolar; a perspectiva de tempo futuro longa, mais do que a de tempo curto, influencia positivamente os esforços dos alunos nas tarefas presentes; a atitude positiva em relação ao próprio futuro é variável relevante para a percepção de instrumentalidade.

Desta forma, com o objetivo geral de compreender tanto aspectos motivacionais de adolescentes brasileiros na escola como sua perspectiva de tempo futuro e a relação entre ambos, o presente estudo exploratório propôs-se responder a quatro questões:

1. Adolescentes da terceira série do Ensino Médio revelam já haver chegado a uma definição vocacional, ou seja, têm uma perspectiva definida de tempo futuro?

2. Qual o grau de motivação e de uso de estratégias pessoais de aprendizagem, por parte destes adolescentes na escola, e como essas variáveis aparecem relacionadas com a escolha vocacional?

3. A escolarização é percebida pelos adolescentes como válida para atingir seus projetos futuros, ou seja, ela tem valor de instrumentalidade para a conquista de suas metas profissionais?

4. A perspectiva de futuro, ou seja, a definição vocacional, prediz a orientação motivacional à meta para aprender, as estratégias de aprendizagem e a percepção de instrumentalidade?

Ao buscar responder a cada uma destas questões, o presente estudo poderá trazer novos conhecimentos sobre o adolescente brasileiro em relação com a escolaridade, sua motivação acadêmica, bem como sobre a relação entre a motivação para estudar e seu momento de escolha vocacional. Como observaram Wigfield e Eccles (2000), ao traçarem linhas possíveis de estudos futuros sobre sua teoria de expectativa-valor, é preciso, entre outras coisas, identificar com mais precisão as relações entre as motivações atuais dos alunos e sua perspectiva de tempo futuro e com os resultados acadêmicos.

\section{Método}

\section{Participantes}

A amostra foi formada por 206 alunos do $3^{\circ}$ ano do ensino médio, na região do norte do Paraná, sendo 88 do sexo masculino (42,71\%) e 118 do feminino (57,28\%). Desse total, 82 estudavam em uma escola particular e os outros 124 em uma escola pública estadual. Além disso, em cada escola havia turnos matutino e noturno, entre os quais se distribuíam os alunos da presente pesquisa, em proporção próxima de $50 \%$ em cada turno. A idade cronológica variou de 15 anos até 19 anos, com prevalência da faixa entre 17 anos e 18 anos e 11 meses (49,5\%). 


\section{Medidas}

Utilizou-se como instrumento para esta pesquisa um questionário de auto-relato com escala tipo Likert, elaborado por Locatelli, Bzuneck e Guimarães (2004). Quatro questões iniciais referiam-se à definição profissional por parte do aluno: se já sabe o que quer ser no futuro (alternativas sim, não, mais ou menos); firmeza ou dúvida quanto a essa decisão (inseguro, pensando pouco nisso, pensando muito nisso, bem definido); e a importância pessoal da carreira escolhida (realizar-se pessoalmente, ganhar bastante dinheiro, poder ter bastante lazer, contribuir com a sociedade, ser útil para as pessoas, ajudar minha família. Outros 34 itens, adaptados de instrumentos já existentes (Cretens, Lens \& Simons, 2001; Husman \& Lens, 1999; Lens, Simons \& Dewitte, 2002; Malka. \& Covington, 2005; Miller \& Brickman, 2004; Simons, Vansteenkiste, Lens \& Lacante, 2004) ou criados para esta pesquisa, destinavamse a avaliar a motivação para estudar, incluindo tempo e esforço empregados em estratégias eficazes, a própria valorização dos estudos no presente e a percepção de que as aprendizagens atuais têm valor de instrumentalidade para a consecução das metas de vida.

Em relação aos 34 itens do questionário, suas propriedades psicométricas foram avaliadas com base na análise dos componetes principais e no levantamento da consistência interna da escala, mediante o alpha de Cronbach. A análise dos componentes principais identificou inicialmente oito fatores com valor próprio acima de 1,00. Adotaram-se, porém, apenas os três primeiros por corresponderem às três subescalas com relevância teórica e que explicam 40,23\% do grau de variância dos dados. Dois itens do questionário original foram excluídos por não atingirem o valor de corte de 0,30. Portanto, foram consideradas para análise as respostas dos participantes para 32 questões, que carregaram em três fatores.

O Fator 1, que passa a ser denominado subescala de avaliação da "motivação", inclui 13 questões que avaliam o quanto de motivação os alunos têm para os estudos, a dedicação, o empenho e o desempenho nas tarefas acadêmicas. $\mathrm{O}$ índice de consistência interna pelo alpha de Cronbach foi de 0,87 .

$\mathrm{O}$ Fator 2 corresponde à subescala que passou a denominar-se "estratégias pessoais de estudo" e verifica o grau de esforço dos alunos nos estudos, o gerenciamento do tempo, além da forma de lidar com a pressão das provas acadêmicas. Nele carregaram 11 itens, com alpha de Cronbach $=0,81$.

Já o Fator 3 foi denominado subescala de avaliação da "percepção de instrumentalidade" e avalia o quanto o aluno percebe os estudos como meios para chegar às suas metas de profissão futura, ou seja, qual é a valorização em termos de utilidade que ele identifica nos estudos de hoje. Oito itens, com alpha de Cronbach $=0,79$, formam este conjunto. Para os itens componentes do instrumento total foi encontrado um índice de consistência interna (alfa de Cronbach) de 0,89.
Os itens do questionário relativos a cada uma dessas três variáveis - motivação, estratégias e percepção de instrumentalidade - eram precedidos de uma questão específica em que o aluno devia apontar uma ou mais, entre as matérias que estuda, que mais serão úteis para sua profissão no futuro. Assim, o auto-relato de motivação, uso de estratégias e percepção de instrumentalidade se referia explicitamente à(s) disciplina(s) apontada(s).

\section{Procedimentos}

O instrumento foi aplicado pela primeira autora nas próprias salas de aula de ambas as escolas e em períodos normais de aula. Aos alunos foi esclarecido o propósito da pesquisa e, além de outras orientações para preenchimento, que a participação era voluntária. Não houve nenhuma recusa em todas as quatro classes, mas, alunos e professores solicitaram um retorno com informações dos resultados obtidos. O tempo médio de aplicação foi de 20 minutos em cada sala das duas escolas.

\section{Resultados}

\section{Identificação dos Alunos em Função da Definição Vocacional}

Procedeu-se, antes de tudo, a uma análise de variância entre os escores médios das quatro turmas da amostra total nas três subescalas em que foram avaliadas: motivação, de estratégias pessoais de estudo e percepção de instrumentalidade, ou seja, buscaram-se aqui as diferenças entre o desempenho dos participantes nas escalas de avaliação e as escolas e os turnos nos quais estudavam. Trabalhou-se, portanto, com uma fatorial 3 x 4 (três variáveis e quatro grupos). Os resultados da análise revelaram algumas diferenças significativas, seja em função da variável, seja em função dos grupos. Não houve, porém, diferenças significativas consistentes nas comparações. Por essa razão, optou-se por tratar as quatro turmas de adolescentes como um grupo único $(N=206)$ para as análises estatísticas seguintes.

O passo seguinte foi verificar, por meio das respostas às quatro questões iniciais do instrumento, se os adolescentes participantes apresentavam-se definidos vocacionalmente, ou seja, uma perspectiva definida de tempo futuro. A condição para que um aluno fosse incluído no grupo chamado "definidos vocacionalmente" era que tivesse respondido às quatro questões da seguinte forma, acumulativamente: (a) ter respondido "sim" na questão 2 (sabe o que quer ser no futuro); (b) na questão 4, que verifica o grau de firmeza quanto à definição vocacional, ter marcado 3 ou 4 (pensando muito ou bem definido); (c) na questão 5 , sobre o grau de indecisão, ter marcado as opções 4 ou 5 (bastante ou totalmente verdadeiro, considerada a pontuação invertida); e (d) ter marcado as opções 1 ou 2 (muito ou bastante importante) na questão 7 , que verifica o grau de importância da profissão. Estas quatro questões selecionadas para compor este critério $(2,4,5$ e 7$)$, foram submetidas a uma análise de 
consistência interna pelo alpha de Cronbach, para verificar o grau de homogeneidade entre os respectivos itens, tendo surgido um valor de $a=0,46$, que sugere não ser adequado trabalhar com seus escores de forma combinada. Por esta razão, em todas as comparações estatísticas seguintes e que envolveram a definição vocacional, aquelas questões foram consideradas separadamente, como já aparece na Tabela 1.
Com base no critério adotado para se estabelecer a definição vocacional, do total da amostra de 206 identificaramse 65 adolescentes, ou 31,5\% (32 da Escola A e 33 da Escola B), que passaram a ser denominados "definidos vocacionalmente". Em contrapartida, os demais 141 alunos, $64,5 \%$ do total, foram considerados "não definidos vocacionalmente”, por não haverem atendido em suas res-

Tabela 1

Correlações de Pearson entre as Variáveis de Definição Vocacional, Grau de Motivação, Uso de Estratégias Pessoais de Estudo e a Percepção de Instrumentalidade dos Estudos Atuais ( $N=65)$

\begin{tabular}{|c|c|c|c|c|c|c|}
\hline & Firmeza & $\begin{array}{l}\text { Nível de } \\
\text { decisão }\end{array}$ & Importância & Motivação & $\begin{array}{l}\text { Estratégias } \\
\text { pessoais de } \\
\text { estudo }\end{array}$ & $\begin{array}{l}\text { Percepção de } \\
\text { instrumen- } \\
\text { talidade }\end{array}$ \\
\hline Firmeza & $1, \mathrm{OO}$ & $0,46^{* * *} *$ & 0,10 & 0,10 & 0,19 & 0,05 \\
\hline $\begin{array}{l}\text { Nível de } \\
\text { decisão }\end{array}$ & & 1,00 & 0,05 & 0,15 & $0,28^{*}$ & 0,08 \\
\hline Importância & & & $1, \mathrm{OO}$ & 0,11 & $0,36^{* *}$ & $0,24^{*}$ \\
\hline Motivação & & & & $1, \mathrm{OO}$ & $0,64 * * *$ & $0,51^{* * *}$ \\
\hline $\begin{array}{l}\text { Estratégias } \\
\text { pessoais } \\
\text { de estudo }\end{array}$ & & & & & 1,00 & $0,37^{* *}$ \\
\hline $\begin{array}{l}\text { Percepção de } \\
\text { instrumen- } \\
\text { talidade }\end{array}$ & & & & & & 1,00 \\
\hline
\end{tabular}

Notas. $* \mathrm{p}<0,01 ;{ }^{*} * \mathrm{p}<0,001 ; * * * \mathrm{p}<0,0001$.

postas a alguma das exigências que compunham o critério adotado. Deste total, 50 alunos são da Escola A $(35,4 \%)$ e 91 da Escola B (64,5\%). Vale acrescentar o dado de que $86,15 \%$ dos alunos definidos e $71,63 \%$ dos não definidos declararam que pretendem prestar vestibular.

\section{Correlações entre as Variáveis}

Procurou-se descobrir as associações entre as variáveis relativas à definição vocacional (firmeza, nível de decisão e importância pessoal da carreira escolhida) e o grau de motivação, uso de estratégias pessoais de estudo e a percepção de instrumentalidade dos estudos atuais. Os resultados das relações entre tais variáveis aparecem na Tabela 1. Observe-se que esta análise contemplou apenas o grupo dos definidos vocacionalmente $(N=65)$ sendo que, em relação a esta variável que englobava as questões 2, 4, 5 e 7, eliminou-se a questão 2 porque, por suposto, todos haviam respondido sim, como variável discreta. Foram encontradas correlações positivas e significativas, entre a questão 4 (que verifica o grau de firmeza quanto à definição vocacional) e a questão 5 (que verifica o grau de indecisão quanto ao futuro); entre a questão 5 e os resultados obtidos na subescala de avaliação das estratégias pessoais de estudo; entre a questão 7 (que aponta o grau de importância da profissão escolhida) e os resultados obtidos nas subescalas de avaliação de estratégias pessoais de estudo e da percepção de instrumentalidade.

\section{Comparação Inter-grupos nas Três Variáveis}

Para identificar possíveis diferenças significativas entre os dois grupos, dos definidos e indefinidos, nas três subescalas - motivação, estratégias pessoais de estudo e percepção de instrumentalidade - foi utilizado o teste $t$ de Student, cujos resultados aparecem na Tabela 2 na próxima página. Como se pode constatar, os escores médios nas três variáveis são mais altos no grupo dos definidos. Porém, a 
diferença é estatisticamente significativa apenas na variável percepção de instrumentalidade.

\section{Análise de Regressão}

$\mathrm{O}$ último objetivo desta pesquisa foi identificar se a definição vocacional é preditiva dos comportamentos referentes à motivação, ao uso de estratégias pessoais de estudo, e da percepção de instrumentalidade. A Análise de Regressão Múltipla foi o teste escolhido para aferir a contribuição relativa de elementos contidos no instrumento para explicar a variância das três variáveis dependentes. Da variável independente definição vocacional foram considerados separadamente os três componentes, avaliados por questões específicas: o grau de firmeza, a decisão e a importância da carreira escolhida. Os dados aparecem na Tabela 3.
Assim, quando se contempla a motivação como variável dependente, nenhum dos componentes da definição vocacional representou valor de predição em nível significativo, isto é, os escores de motivação não aparecem com característica de dependência em relação à firmeza da escolha, nem ao grau de decisão e nem à importância dada à escolha vocacional.

Quanto à variável dependente estratégias pessoais de estudo, apareceu efeito preditivo positivo, em nível significativo, por parte do grau de decisão do que o aluno quer ser no futuro. Para a mesma variável dependente, a importância de se chegar à profissão escolhida foi preditiva em nível significativo $(p=0,003)$ e grau moderado. Desse modo, considerar a importância da profissão futura influencia direta e significativamente na forma como os alunos da amostra estudam e organizam seu tempo.

Tabela 2

Comparação entre os Grupos dos Definidos e dos Não Definidos Vocacionalmente, nas Variáveis Motivação, Estratégias Pessoais de Estudo e Percepção de Instrumentalidade

\begin{tabular}{|c|c|c|c|c|c|c|c|c|c|}
\hline & \multicolumn{3}{|c|}{ Definidos } & \multicolumn{3}{|c|}{ Não definidos } & \multirow[b]{2}{*}{$\mathrm{t}$} & \multirow[b]{2}{*}{$\mathrm{p}$} & \multirow[b]{2}{*}{$\mathrm{gl}$} \\
\hline & $\mathrm{N}$ & M & $\mathrm{Dp}$ & $\mathrm{N}$ & M & $\mathrm{Dp}$ & & & \\
\hline Motivação & 63 & 3,00 & 0,87 & 134 & 2,94 & 0,73 & $-0,54$ & 0,59 & 195 \\
\hline $\begin{array}{l}\text { Estratégias pes- } \\
\text { soais de estudo }\end{array}$ & 64 & 3,46 & 0,85 & 132 & 3,28 & 0,73 & $-1,49$ & 0,14 & 194 \\
\hline $\begin{array}{l}\text { Percepção de ins- } \\
\text { trumentalidade }\end{array}$ & 64 & 4,10 & 0,58 & 132 & 3,87 & 0,68 & $-2,39$ & $0,02^{*}$ & 194 \\
\hline
\end{tabular}

Tabela 3

Análise de Regressão de cada Variável Dependente (V.D.) para cada Componente da Definição Vocacional, como Variável Independente (V.I.)

$\begin{array}{llllll}\text { BETA } & \text { d.p. } & \text { B } & \text { d.p. } & \mathrm{t}(59) & \text { p }\end{array}$

V.D.: Motivação

V.I.:

Firmeza

0,02

O, 14

0,05

0,25

0,20

ns

Decisão

o,13

0,14

0,30

0,95

ns

Importância

$$
0,10
$$

0,12

0,23

0,29

0,78

ns

V.D.: Estratégias

V.I.:

Firmeza

0,03

0,27

O, 13

0,05

0,22

0,23

ns

Decisão

0,35

0,12

0,56

0,24

2,29

0,02

Importância

o,79

0,26

2,99

0,003

V.D.: Percepção de instrumentalidade

V.I.:

Firmeza

Decisão

Importância

$\begin{array}{cccccc}0,001 & 0,14 & 0,001 & 0,16 & 0,01 & \text { ns } \\ 0,07 & 0,14 & 0,09 & 0,19 & 0,49 & \text { ns } \\ 0,24 & 0,12 & 0,36 & 0,18 & 1,96 & 0,05\end{array}$


Por último, quando se considerou como variável dependente a percepção de instrumentalidade por parte dos alunos, o método de regressão revelou que teve valor de predição em nível significativo somente a questão relativa à importância de se alcançar a profissão pretendida no futuro. Vale observar que, pela análise de regressão, o grau de firmeza quanto a estar definido para a profissão futura não foi, isoladamente, preditivo de nenhuma das três variáveis dependentes.

\section{Discussão}

Este trabalho objetivou responder a diversas questões que convergiam para uma melhor compreensão da motivação de adolescentes brasileiros na escola, especificamente em relação com a perspectiva de tempo futuro ou escolha vocacional. Pelo final do ensino médio, espera-se que os adolescentes estejam definindo sua identidade vocacional, especialmente se se considera a iminência de um possível vestibular. Assim, a primeira descoberta do presente estudo que merece comentário diz respeito à escolha vocacional, ou seja, se aqueles adolescentes já têm um projeto de vida definido em termos profissionais.

Ora, os dados revelam que pouco menos de um terço da amostra total se declarou definido vocacionalmente e com segurança de sua escolha. A proporção dos indefinidos é bem maior entre os alunos da escola pública. Por um lado, isto é surpreendente, considerando-se a proximidade do término do ensino médio e o possível ingresso em curso superior mediante vestibular. Aliás, mais de dois terços de cada grupo da amostra total mostraram-se inclinados a prestar vestibular. Entretanto, ao menos no que se refere a adolescentes brasileiros, a indecisão vocacional nessa fase não foi uma descoberta isolada. Embora em alguns casos com amostras reduzidas, outros estudos brasileiros revelaram a mesma tendência, como os de Günther e Günther (1998), Oliveira, Souza, Pinto e Oliveira Júnior (2002), Sarriera, Silva, Kabbas e Lopes (2001), Schoen-Ferreira, Aznar-Farias e Silvares (2003). E, tanto no presente como nos estudos desses autores, a indefinição atinge sobremaneira os alunos de escolas públicas e de nível sócio-econômico inferior. Por exemplo, Sarriera et al. (2001) haviam constatado que os adolescentes de sua amostra apresentavam dúvidas quanto a suas escolhas vocacionais por não saberem o que podiam esperar de seu futuro, independentemente da definição quanto ao vestibular.

Erikson (1971), embora tenha considerado as dificuldades reais que caracterizam a escolha vocacional na adolescência, supunha que essa é a fase certa para a definição. Mais recentemente, porém, Steinberg e Morris (2001), após revisarem a literatura que considerou as condições atuais, concluíram que hoje a formação da identidade vocacional não deveria mais ser esperada até aos 18 anos, como supunha Erikson (1971), mas pode ser estendida para mais tarde, quando o jovem tenha conseguido ponderar todas as instâncias pertinentes.

Daí se depreende não ser realístico que professores usem indiscriminadamente para todos, como recurso motivacional, apelar para o valor de utilidade de certo conteúdo de aprendizagem atual para profissões futuras. Os dados de pesquisa sugerem que para a maioria dos adolescentes do ensino regular essa alegação não faz muito sentido e, portanto, não os animaria a estudar mais. A previsão é de ser diferente a condição dos alunos de escolas profissionalizantes ou dos cursinhos pré-vestibulares.

\section{Relação entre a Definição Vocacional e as Variáveis Dependentes}

$\mathrm{O}$ foco principal de investigação do presente estudo foram as variáveis grau de motivação, uso de estratégias de aprendizagem e percepção de instrumentalidade, e se elas têm relações significativas com metas futuras. Mais ainda, buscou-se verificar se a perspectiva de tempo futuro seria preditiva da motivação, do uso de estratégias pessoais de estudo, além da percepção de instrumentalidade. Para isso compararam-se os dois grupos - dos definidos e dos não definidos vocacionalmente, ou seja, dos que têm e dos que não têm uma perspectiva de tempo futura estabelecida.

As médias em cada variável foram mais altas no grupo dos definidos vocacionalmente que as obtidas pelo grupo não definido. Em outras palavras, os alunos do primeiro grupo revelam uma tendência de serem mais motivados para estudar, a apresentarem estratégias pessoais de estudo mais adequadas às exigências escolares, e a terem uma maior percepção da importância dos estudos no presente para alcançarem suas metas colocadas no futuro. Entretanto, foi estatisticamente significativa a diferença das médias, a favor do grupo dos definidos vocacionalmente, apenas na variável percepção de instrumentalidade. Isto é, na motivação e uso de estratégias os grupos se eqüivalem, conforme o teste estatístico. Todos esses resultados merecem consideração especial, por se referirem ao âmago da motivação por metas futuras.

Em primeiro lugar, faz sentido que os alunos com definição de metas futuras também percebam mais a função dos estudos como meios para as atingirem, ou seja, trabalham com um esquema chamado meios-fins. A meta futura é pessoalmente valorizada e assim é incentivo para o envolvimento com os estudos, que são agora valorizados pelo seu aspecto de utilidade (Wigfield \& Eccles, 2000). A análise de regressão, aplicada aos dados do presente estudo, confirma que a meta futura tem valor preditivo em relação às ações que, de modo instrumental, conduzam àquela meta, ou seja, aí se configura a motivação instrumental (Lens et al., 2002). Assim, muitos adolescentes podem esmerar-se por aprender, tirar boas notas, entre outros objetivos, porque aspiram prosseguir nos estudos visando uma determinada profissão na vida adulta. Para eles a escolaridade é orientada para o seu futuro e, para isso, o fator crucial é que tenham estabelecidos para si próprios tais projetos de vida. Assim como no presente estudo, a literatura tem comprovado essa relação de forma consistente: Cretens et al. (2001), De Volder e Lens (1982), Greene et al. (2004), Husman e Lens, (1999); Lens et al. (2002) e Van Calster et al. (1987). Por último, os dados do presente estudo mostraram também que a percepção de instrumentalidade 
por parte dos alunos esteve mais associada à preferência por estratégias de aprendizagem chamadas significativas ou de profundidade. Por meio delas o aluno busca compreensão genuína dos conteúdos, mediante relação com conhecimentos prévios e a organização como métodos de estudo. Isto significa que a qualidade do investimento de tempo e esforço, em termos de preferência por esse tipo de estratégias, está significativamente associada às percepções de que as aprendizagens no presente são meios para a consecução de metas de vida (ver também Brickman \& Miller, 2001; Miller \& Brickman, 2004).

Resta explicar a descoberta não esperada de que o estabelecimento de metas profissionais futuras não apareceu como preditiva das variáveis motivação e uso de estratégias pessoais de estudo. E as correlações nos dois casos foram em grau apenas discreto. Nessas duas variáveis, também não foi significativa a diferença entre os escores médios dos dois grupos, embora os dos definidos sejam mais altos. Também entre os alunos deste último grupo os escores em motivação se relacionavam positivamente, em grau significativo, com o uso de estratégias e com percepção de instrumentalidade. Que escores em motivação e em uso de estratégias adequadas de aprendizagem estejam positivamente relacionados é um dado amplamente comprovado por pesquisas (ver, por exemplo, Greene et al., 2004 e a revisão de Bzuneck, 2005, com alunos brasileiros de cursos superiores).

O grupo dos definidos mostrou-se motivado, embora o outro também o fosse. Porém, de qual motivação se trata? Lens et al. (2002) ponderam que nos casos de motivação instrumental, característica do primeiro grupo, as aprendizagens escolares podem ser abraçadas por motivação extrínseca, que seriam as metas futuras. A motivação extrínseca se configura quando a atividade não é exercida como um fim em si mesmo, mas por razões externas a ela, como notas, recompensas, evitar punições, conseguir benefícios futuros etc. É razoável a suposição de que os alunos do ensino médio carreguem uma longa história de motivação prevalentemente extrínseca nas aprendizagens escolares, o que afetaria igualmente, na presente amostra, tanto os definidos como os não definidos. Daí sua semelhança de escores no auto-relato na escala de motivação. Os itens do presente questionário de motivação podem ainda ter contribuído para esse resultado, de modo que outros instrumentos de medida de motivação presumivelmente propiciariam outros resultados.

Quanto ao uso de estratégias pessoais de estudo, a semelhança nos resultados dos dois grupos pode ser igualmente explicada por limitações do instrumento utilizado, que privilegiou apenas algumas poucas estratégias, salientandose a de gerenciamento do tempo para estudo e preparação de provas. Mesmo assim, deve-se ter presente que o conhecimento e uso de estratégias eficazes de aprendizagem supõem orientações explícitas por parte dos professores (Boruchovitch, 1999; Wood, Motz \& Willoughby, 1998). Pode-se até supor que, em relação aos alunos da amostra inteira, não importando a distinção dos dois grupos ora em foco, pouco teria sido feito em termos de ensino e treina- mento de estratégias eficazes de estudo, o que levou à equiparação dos resultados nessa variável.

Em síntese, a definição vocacional, ou meta futura, revelou-se como variável importante na escolaridade do adolescente ao final do ensino médio. Em que pese o fato de não se ter trabalhado com uma amostra muito numerosa, o que é uma limitação do presente estudo, as descobertas lançam alguma luz sobre a motivação dos adolescentes. Os professores em sala de aula podem mostrar o valor de utilidade de determinadas disciplinas mostrando que elas representam meios para a conquista de metas futuras, quando já definidas ou em fase de definição. Entretanto, a motivação instrumental não prescinde de outras estratégias, descritas por autores como Bandura e Schunk (1981), Guimarães (2004) e Hidi e Ainley (2002), que exploram diretamente as tarefas acadêmicas do dia-a-dia. Características de serem próximas e desafiadoras, seguidas do competente feedback, têm o potencial de fazer o aluno chegar a valorizar as aprendizagens escolares, atingindo eventualmente o nível da motivação intrínseca, motivação extrínseca auto-regulada ou da orientação prevalente à meta de realização aprender.

\section{Referências}

Bandura, A. (1997). Self-efficacy: The exercise of control. New York: W. H. Freeman.

Bandura, A., \& Schunk, D. H. (1981). Cultivating competence, selfefficacy, and intrinsic interest through proximal self-motivation. Journal of Personality and Social Psychology, 41, 586-598.

Boruchovitch, E. (1999). Estratégias de aprendizagem e desempenho escolar: Considerações para a prática escolar. Psicologia: Reflexão e Crítica, 12, 361-373.

Brickman, S. J., \& Miller, R. B. (2001).The impact of sociocultural context on future goals and self-regulation. In D. M. McInerney \& S. Van Etten (Eds.), Research on sociocultural in-fluences on motivation and learning (pp. 119 -137). Greenwich, CT: Information Age.

Bzuneck, J. A. (2005). A motivação dos alunos em cursos superiores. In M. C. R. A. Joly, A. A. A. dos Santos \& F. F. Sisto (Eds.), Questões do cotidiano universitário (pp. 217-237). São Paulo, SP: Casa do Psicólogo.

Bzuneck, J. A. \& Boruchovitch, E. (2003). Adolescence and education: Contemporary trends in brazilian research. In F. Pajares \& T. Urdan (Eds.), International perspectives on adolescence (pp. 215-236). Greenwich, CT: Information Age.

Cretens, H., Lens, W., \& Simons, J. (2001). The role of perceived instrumentality in student motivation. In A. Anastasia, J. Kuhl \& R. M. Sorrentino (Eds.), Trends and prospects in motivation research (pp. 37-45). Dordrecht, Netherlands: Kluwer Academic.

Deci, E. L., \& Ryan, R. M. (1985). Intrinsic motivation and selfdetermination in human behavior. New York: Plenum Press.

De Volder, M. L., \& Lens, W. (1982). Academic achievement and future time perspective as a cognitive-motivational concept. Journal of Educational Psychology, 42, 566-571.

Eccles, J. S., \& Wigfield, A. (2002). Motivational beliefs, values, and goals: Learning and performance in educational settings. Annual Review of Psychology, 53, 109-132.

Erikson, E. H. (1971). Infância e sociedade. Rio de Janeiro, RJ: Jorge Zahar. 
Greene, B. A., Miller, R. B., Crowson, H. M., Duke, B. L., \& Akey, K. L. (2004). Predicting high school students cognitive engagement and achievement: Contributions of classroom perceptions and motivation. Contemporary Educational Psychology, 29, 462-482.

Guimarães, S. E. R. (2004). A organização da escola e da sala de aula como determinante da motivação intrínseca e da meta aprender. In E. Boruchovitch \& J. A. Bzuneck (Eds.), A motivação do aluno: Contribuições da Psicologia Contemporânea (pp. 78-95). Petrópolis, RJ: Vozes.

Günther, I. A., \& Günther, H. (1998). Brasílias pobres, Brasílias ricas: Perspectivas de futuro entre adolescentes. Psicologia: Reflexão e Crítica, 11, 191-207.

Hidi, S., \& Ainley, M. (2002). Interest and adolescence. In F. Pajares \& T. Urdan (Eds.), Academic motivation of adolescents (pp. 247-275). Greenwich, CT: Information Age.

Husman, J., \& Lens, W. (1999). The role of the future in student motivation. Educational Psychologist, 34, 113-125.

Lens, W., \& Decruynaere, M. (1991). Motivação e desmotivação no ensino secundário: As características dos alunos. Psychologica, 6, 13-31.

Lens, W., Simons, J., \& Dewitte, S. (2002). From duty to desire: The role of students' future time perspective and instrumental perceptions for study motivation and self-regulation. In F. Pajares \& T. Urdan (Eds.), Academic motivation of adolescents (pp. 22 1-245). Greenwich, CT: Information Age.

Locatelli, A. C. D., Bzuneck, J. A., \& Guimarães, S. E. R. (2004). Questionário de motivação e metas futuras. Londrina: Universidade Estadual de Londrina. Manuscrito não publicado.

Malka, A., \& Covington, M. V.(2005). Perceiving school performance as instrumental to future attainment: Effects on graded performance. Contemporary Educational Psychology, 30, 61-80.

Markus, H., \& Nurius, P. (1986). Possible selves. American Psychologist, 41, 954-969.

Miller, R. B., \& Brickman, S. A. (2004). A model of future oriented motivation and self-regulation. Educational Psychology Review, 16, 9-33.

Oliveira, M. C., Souza, A. S., Pinto, R. G., \& Oliveira, A. M. L., Jr. (2002). Expectativas quanto ao ingresso na Universidade entre adolescentes de diferentes inserções geográficas do Distrito Federal. Trabalho apresentado na XXXII Reunião Anual de Psicologia. Florianópolis, SC: Sociedade Brasileira de Psicologia.

Pintrich, P.R. (2000). Achievement goal theory perspective in motivation and terminology, theory, and research. Contemporary Educational Psychology, 25, 92-104.

Ryan, R. M., \& Deci, E. L. (2000). Intrinsic and extrinsic motivations: Classics definitions and new directions. Contemporary Educational Psychology, 25, 54-67.
Sarriera, J. C., Silva, M. A., Kabbas, C. P., \& Lópes, V. B. (2001). Formação da identidade ocupacional em adolescentes. Estudos de Psicologia, 6, 16-32.

Schoen-Ferreira, T. H., Aznar-Farias, M., \& Silvares, E. F. M. (2003). A construção da identidade em adolescentes: Um estudo exploratório. Estudos de Psicologia, 8, 107-115.

Shell, D. F., \& Husman, J. (2001). The multivariate dimensionality of personal control and future time perspective beliefs in achievement and self-regulation. Contemporary Educational Psychology, 26, 481-506.

Simons, J., Vansteenkiste, M., Lens, W., \& Lacante, M. (2004). Placing motivational and future time perspective theory in a temporal perspective. Educational Psychology Review, 16, 121139.

Steinberg, L., \& Morris, A. S. (2001). Adolescent development. Annual Review of Psychology, 52, 83-110.

Van Calster, K., Lens, W., \& Nuttin, J. R. (1987) Affective attitude toward the personal future: Impact on motivation in high school boys. American Journal of Psychology, 100, 1-13.

Weiner, B. (1984). Principles for a theory of student motivation and their application within an attributional framework. In R. Ames \& C. Ames (Eds.), Research on motivation in Education: Vol. 1. (pp. 15-38). New York: Academic Press.

Weiner, B. (2004). Attribution theory revisited: Transforming cultural plurality into theoretical unity. In D. M. McInerney \& S. Van Etten (Eds.), Big theories revisited (pp. 13-29). Greenwich, CT: Information Age.

Wigfield, A., \& Eccles, J. S. (2000). Expectancy-value Theory of Achievement Motivation. Contemporary Educational Psychology, 25, 68-81.

Wigfield, A., Tonks, S., \& Eccles, J. S. (2004). Expectancy-value Theory in Cross-Cultural Perspective. In D. M. McInerney \& S. Van Etten (Eds.), Big theories revisited (pp. 165-198). Greenwich, CT: Information Age.

Wood, E., Motz, A., \& Willoughby, T. (1998). Examining students' retrospective memories of strategy development. Journal of Educational Psychology, 90, 698-704.

Zusho, A., \& Pintrich, P. R. (2001). Motivation in the second decade of life: The role of multiple developmental trajectories. In T. Urdan \& F. Pajares (Eds.), Adolescence and education: General issues in the education of adolescents (pp. 163-200). Greenwich, CT: Information Age.

Recebido: 20/07/2005 $1^{a}$ revisão: $26 / 07 / 2006$ Aceite final: 23/10/2006 